Supporting Information

\title{
A Versatile Theranostic Delivery Platform Integrating Magnetic Resonance Imaging/Computed Tomography, pH/cis-Diol Controlled Release and Target- ed Therapy
}

Yu-Jui Tseng, ${ }^{\dagger}$ Shang-Wei Chou, ${ }^{\dagger}$ Jing-Jong Shyue, ${ }^{\# \perp}$ Shih-Yao Lin, ${ }^{\dagger}$ Jong-Kai Hsiao, ${ }^{*,+\S}$ Pi-Tai Chou* ${ }^{*}$

\footnotetext{
${ }^{\dagger}$ Department of Chemistry, National Taiwan University, Taipei 10617, Taiwan

"Department of Medical Imaging, Taipei TzuChi Hospital, The Buddhist Tzuchi Medical foundation, Taipei 23142, Taiwan \#Department of Materials Science and Engineering National Taiwan University, Taipei 10617, Taiwan

$\perp$ Research Center for Applied Science, Academia Sinica, Taipei 11529, Taiwan

${ }^{\S}$ School of Medicine, Tzu-Chi University, Hualien 97004, Taiwan
}

*E-mail address: chop@ntu.edu.tw; jongkai@tzuchi.com.tw 


\section{Table of Contents}

Experimental Section

Figure S1. TEM images of extremely small iron oxide nanoparticles (ESIONPs).

Figure S2. TEM images of synthesis of mesoporous silica coated ESIONPs without core. ------------------------------5

Figure S3. TEM images of ESIONPs@WMSNs. -----------------------------------------------------------------6

Figure S4. UV-Vis spectrum of the as-synthesized BAuNPs. ----------------------------------------------------------7

Figure 55. (a) SEM images of ligand-mS-ESIONPs@WMSNs. (b) TEM image of BAuNPs-ligand-mS-ESIONPs@WMSN. (c) The HRTEM image of ligand-mS-ESIONPs@WMSN. (d) The HRTEM image of BAuNPs-ligand-mS-

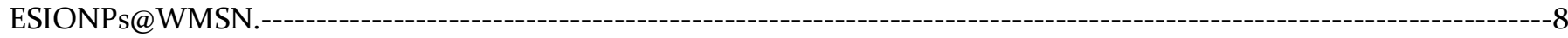

Figure S6. X-ray photoelectron spectroscopy (XPS) of ESIONPs. ---------------------------------------------------------9

Figure S7. The full XPS spectrum of mS-ESIONPs@WMSN and the corresponding detailed spectra of major component elements.

Figure S8. Energy-dispersive X-ray (EDX) spectroscopy analysis. --------------------------------------------------------11

Figure S9. (a) $\mathrm{N}_{2}$ adsorption-desorption analysis (BET). (b) pore-size distribution (BJH). (c) BET surface area and pore size calculated from $\mathrm{N}_{2}$ adsorption-desorption isotherm. $-12$

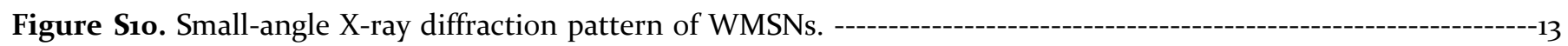

Figure S11. X-ray diffraction spectra.

Figure S12. FT-IR spectra of FA-PCA-BAuNPs-ligand-mS-ESIONPs@WMSNs.

Figure S13. Release profiles of Safranin O dye in water from BAuNPs-ligand-mS-ESIONPs@WMSNs at pH 2-3. ------16

Figure S14. MTT Assay.

Figure S15. (a)Prussian blue stained images. (b) Relaxation properties of the nanocomposites at 0.47 T. (c) T1weighted images of BAuNPs-ligand-mS-ESIONPs@WMSNs.

The estimation of the quantity of nanoparticles per cell-

Figure S16. Serial sections of Z-stack confocal microscopic images. -------------------------------------------------20

Figure S17. The $\mathrm{T}_{1}$-weighted images of FA-PCA-BAuNPs-ligand-mS-ESIONPs@WMSNs were monitored using a $7 \mathrm{~T}$ animal micro MRI system as the NPs were administrated by intravenous injection of $1 \mathrm{mg} \mathrm{Fe} / \mathrm{kg}$. ------- 21

Figure S18. Using the same procedure of adsorbing nanoparticles on the surface of wormlike mesoporous silica and then coated with a thin layer of mesoporous silica shell to encapsulate quantum dots within WMSN. (a) The as-synthesized nanoparticles. (b) Particles under UV lamp excitation source.

Figure S19. Confocal images of control set for HeLa and A549 cell lines without being fed with doxorubicin. ---------23 
Chemicals. Iron chloride $\left(\mathrm{FeCl}_{3} \cdot 6 \mathrm{H}_{2} \mathrm{O}, 98 \%\right.$, Sigma Aldrich), sodium oleate (95\%, TCI), oleyl alcohol (6o\%, Acros), 1-octadecene (ODE, 90\%, Acros), tetraethyl orthosilicate (TEOS, 98\%, Acros), ammonium fluoride $\left(\mathrm{NH}_{4} \mathrm{~F}\right.$, Acros, 98\%), ammonium nitrate (99.0\%, Sigma Aldrich), cetyltrimethylammonium bromide (CTAB, 99\%, Acros), $\mathrm{N}$-(3-triethoxysilylpropyl)gluconamide (50\% in ethanol, ABCR), hydrogen tetrachloroaurate(III) trihydrate $\left(\mathrm{HAuCl}_{4} \cdot \mathrm{H}_{2} \mathrm{O}\right.$, Acros), 4-Mercaptophenylboronic acid (90\%, Sigma Aldrich), 3, 4-dihydroxybenzoic acid (97\%, Alfa Aesar), folic acid (97\%, Sigma Aldrich), Acetic acid (99.8\%, Acros), 1-ethyl-3-(3dimethylaminopropyl)carbodiimide (99\%, Carbosynth), n-hydroxysuccinimide ( $98 \%$, Acros), sodium hydroxide ( $\mathrm{NaOH}, 98 \%$ SHIMAKYU), hydrochloric acid ( $\mathrm{HCl}, 37 \%$, Acros), safranine O (85\%, Sigma Aldrich), doxorubicin hydrochloride (DOX, 97\%, AKSci). All chemicals were used as received without further purification. Deionized water was used in all experiments.

Synthesis of Extremely Small-Sized Iron Oxide Nanoparticles. Iron-oleate complex and extremely smallsized iron oxide nanoparticles were synthesized according to the previously reported procedure with some modifications $^{22}$. For the synthesis of $2 \mathrm{~nm}$-sized iron oxide nanoparticles, $1.8 \mathrm{~g}$ of iron-oleate complex ( $\left.2 \mathrm{mmol}\right), 3.835$ $\mathrm{mL}$ of oleyl alcohol $(12 \mathrm{mmol}$ ) were dissolved in $10 \mathrm{ml}$ of 1-octadecane at room temperature. The reaction mixture was degassed at $110{ }^{\circ} \mathrm{C}$ under vacuum for $2 \mathrm{~h}$. The mixture was then heated to $200{ }^{\circ} \mathrm{C}$ with a constant heating rate of $3.3^{\circ} \mathrm{C} / \mathrm{min}$, and then kept at that temperature for 30 min under nitrogen. The resulting solution containing the nanoparticles was then rapidly cooled to room temperature, and $70 \mathrm{ml}$ of isopropanol was added to the solution to precipitate the nanoparticles. The nanoparticles were separated by centrifugation and dispersed in $10 \mathrm{ml}$ hydrophobic solvent such as hexane or chloroform.

Synthesis of B-AuNPs. The boronic acid functionalized nanoparticles (B-AuNPs) were obtained following a modification of the Brust method. Hydrogen tetrachloroaurate (III) and 4-mercaptophenylboronic acid (o.19 $\mathrm{mmol})$ were dissolved in methanol $(75 \mathrm{~mL})$. To prevent deprotonation of the boronic acid, acetic acid $(1.5 \mathrm{ml})$ was added. $15 \mathrm{ml}$ of freshly prepared $0.4 \mathrm{~mol} \mathrm{dm}^{-3}$ aqueous sodium borohydride solution were then carefully added in small portions of ca. $1 \mathrm{ml}$ with vigorous stirring. The solution turned purple immediately, indicating the formation of gold nanoparticles in the size range of $\sim 3.5 \mathrm{~nm}$. After further stirring for 30 min the solution was centrifuged at $10000 \mathrm{rpm}$ for $10 \mathrm{~min}$ to completely remove the aggregated precipitates, and the supernatants were collected for further preparation.

Relaxivity Measurement. The $r_{1}$ and $r_{2}$ relaxation times were measured at $40{ }^{\circ} \mathrm{C}$ using a $0.47 \mathrm{~T}$ Minispec spectrometer (Bruker Minispec mq series relaxometer); $r_{2}$ relaxation times were determined using a CarrPurceMeiboomGill (CPMG) sequence, with a recycle time $10 \mathrm{sec}$, eight averages with phase cycling, and $180^{\circ}$ pulse separation of $1 \mathrm{~ms}$. Monoexponetial fitting was performed to even echoes over a $250 \mathrm{~ms}$ acquisition window. The $r_{1}$ relaxation was estimated using inversion recovery techniques, with a recycle time of 1o sec, four averages with phase cycling, and eight inversion times logarithmically spaced over the interval o-20oo ms. Linear regression between $r_{1}$ and $r_{2}$ and iron concentration was iron concentration was performed using standard techniques.

Characterizations. The shapes and size distributions of the nanocrystals were measured with a JEOL JEM 1230 transmission taken with a JEOL JEM $2100 \mathrm{~F}$ microscope operated at $100 \mathrm{kV}$. Experiments of X-ray energy dispersive spectroscopy (EDS) were performed by using a GENESIS 2000 EDS detector connected to the HRTEM instrument. The conventional Formvar coated 200 mesh $\mathrm{Cu}$ grids were used for the above-mentioned electron microscope (EM) experiments. Powder X-ray diffraction data was collected on a Bruker D8 Advance diffractometer. The workup procedure was carried out with $\mathrm{Cu} K \gamma$ radiation $(\lambda)$ 1.54178 Å. Magnetic measurements were performed by superconducting quantum interference device (SQUID) magnetometer (MPMS, Quantum Design). The measurements were recorded between -20000 and 20000 Oe at $300 \mathrm{~K}$. The specific surface area of the sample was calculated based on the Brunauer-Emmett-Teller (BET) equation at P/Po of between 0.05 and o.3. The total pore volume was calculated from the branch of the adsorption isotherm using a Barrett-Joyner-Halenda $(\mathrm{BJH})$ method. The XPS experiment was performed on VG Scientific ESCALAB 250. The x-ray source was a monochromatic $\mathrm{Al} \mathrm{K \alpha}$ source $\left(1486.7 \mathrm{~nm}, 15 \mathrm{kV}, 200 \mathrm{~W}\right.$, beam size: $\left.650 \mu \mathrm{m}^{2}\right)$. X-ray incident angle was 47.3 degree and collection angle 90 degree. Energy analyzer was a spherical sector analyzer with multi-channeltron array. The neutralization current was between 2.5 A to 2.9 A, depending on the conductivity of the sample. All data was acquired when the pressure of the analysis chamber was lower than $\sim 10^{-9}$ torr. The sample was adhered to the holder using a carbon conductive tape. UV-Vis spectrometry (HITACHI U-3310) was used to determine the modification of nanoparticles or ligands. 

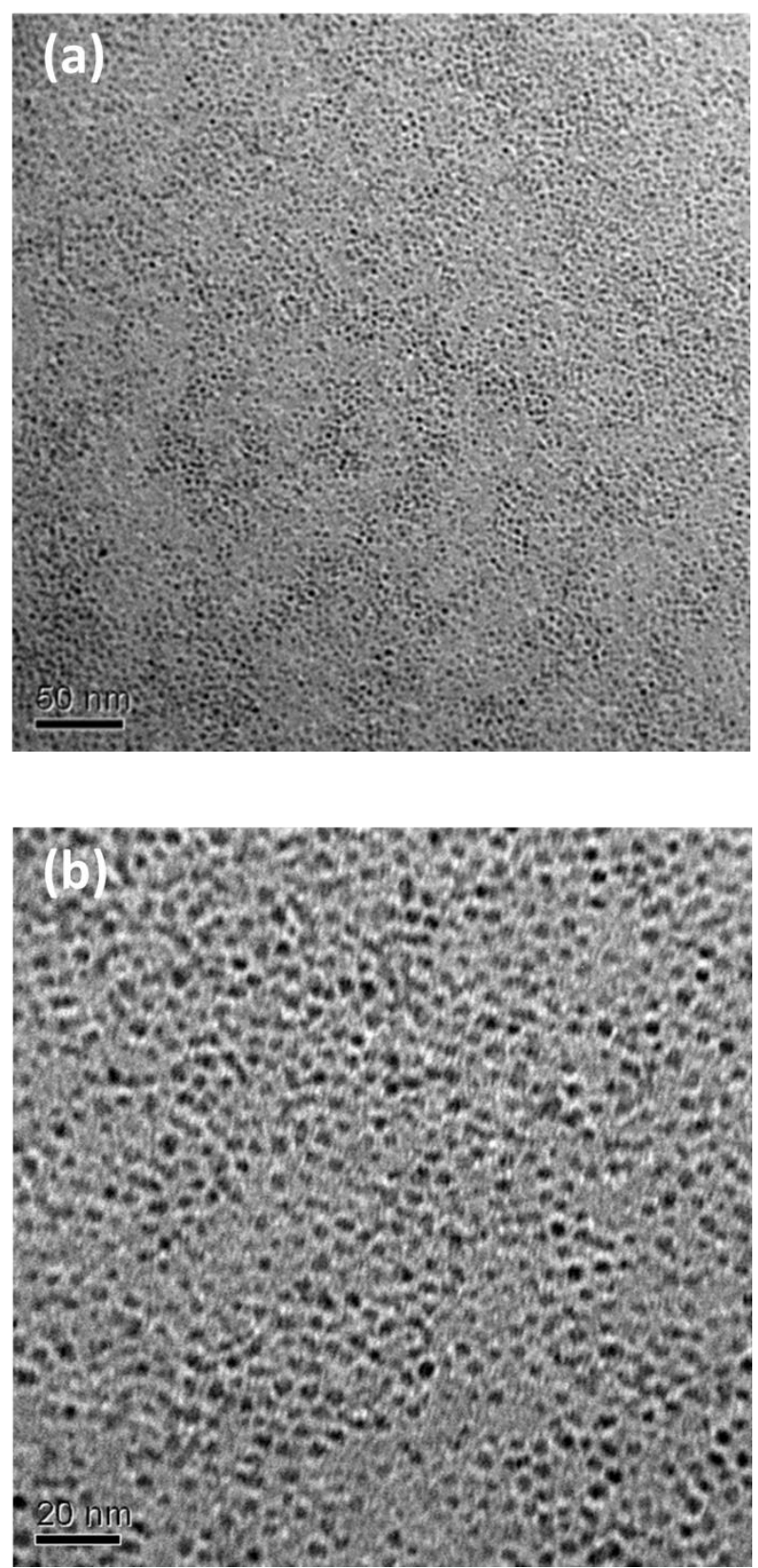

Figure S1. TEM images of extremely small iron oxide nanoparticles (ESIONPs) dispersed in organic phase hexane, (a) low and (b) high magnification. The diameter is about $2 \mathrm{~nm}$. 


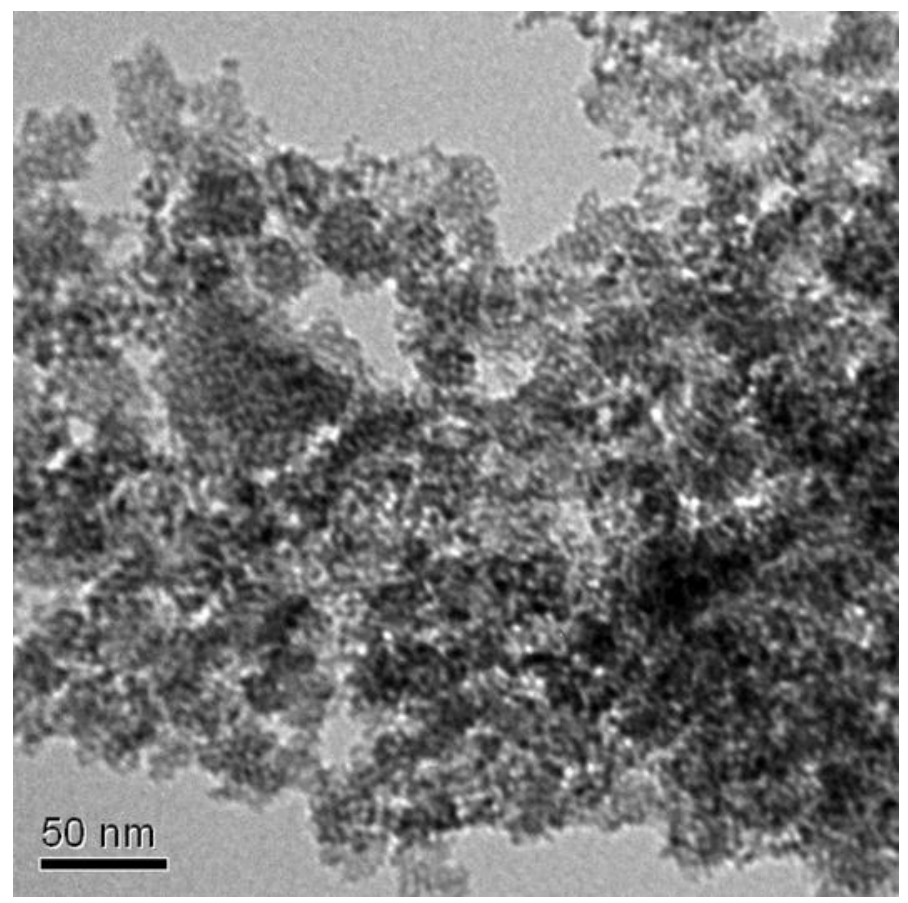

Figure S2. TEM images of synthesis of mesoporous silica coated ESIONPs without core. 


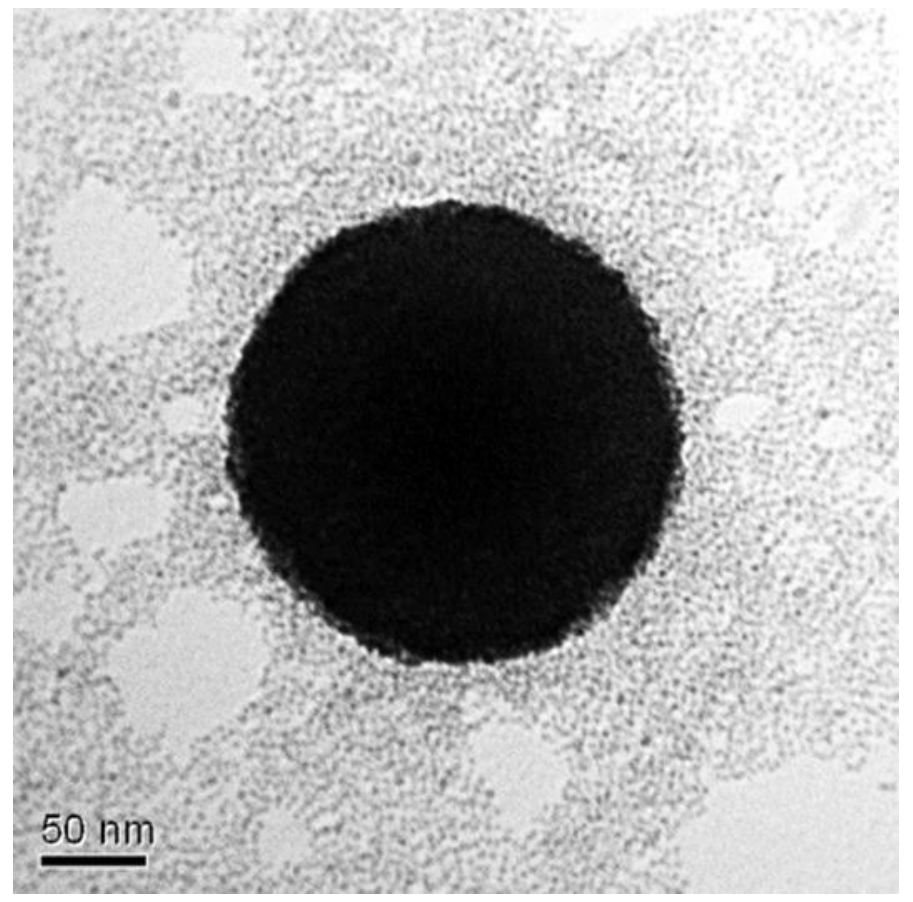

Figure S3. TEM images of ESIONPs@WMSNs. This picture is shown to demonstrate that ESIONPs can adsorb onto WMSNs. We can see from this picture that a large amount of ESIONPs are on the surface of WMSNs. The purpose of using WMSNs up to $150 \mathrm{~nm}$ is to enhance the adsorption of the assynthesized extremely small iron oxide nanoparticles owing to the larger surface area (cf. the size of 100 $\mathrm{nm}$ ) that adsorb more iron oxide nanoparticles. In the bio-related experiments, particle size of mesoporous silica below $100 \mathrm{~nm}$ is favorable for cells or animals to ingest and thus we chose particle size below $100 \mathrm{~nm}$ as our drug delivery system core. 


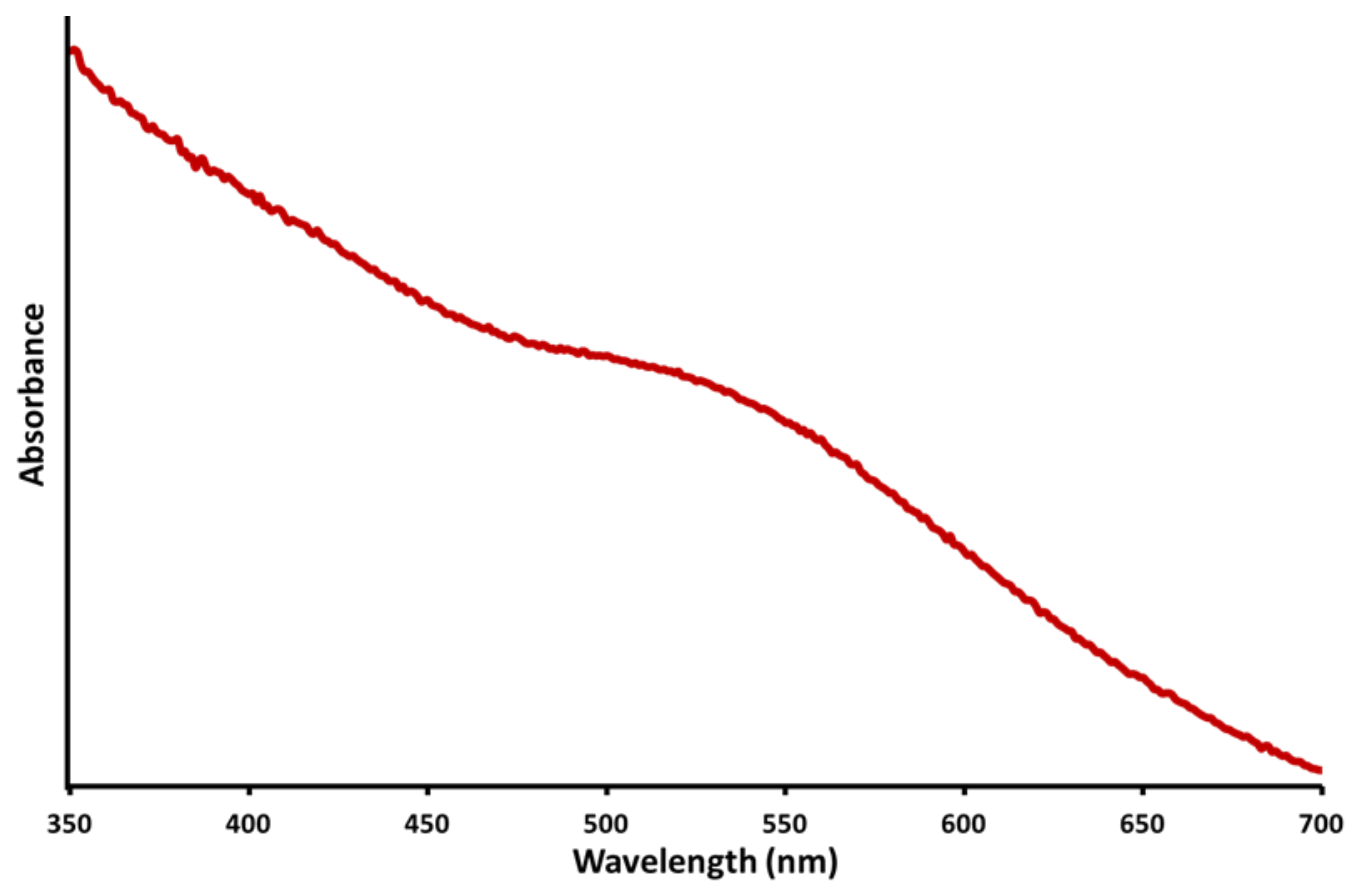

Figure S4. UV-Vis spectrum of the as-synthesized BAuNPs. 
(a)

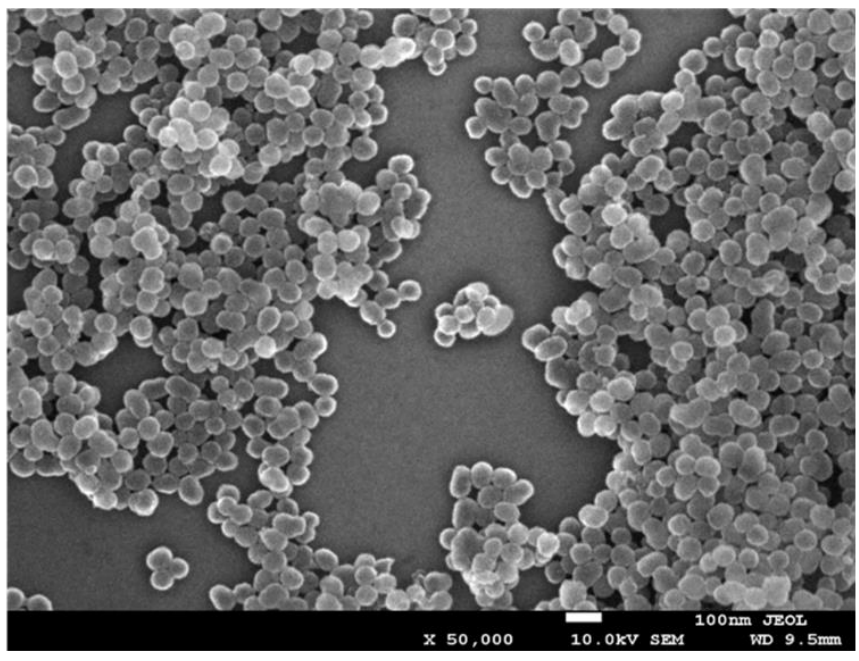

\section{(b)}

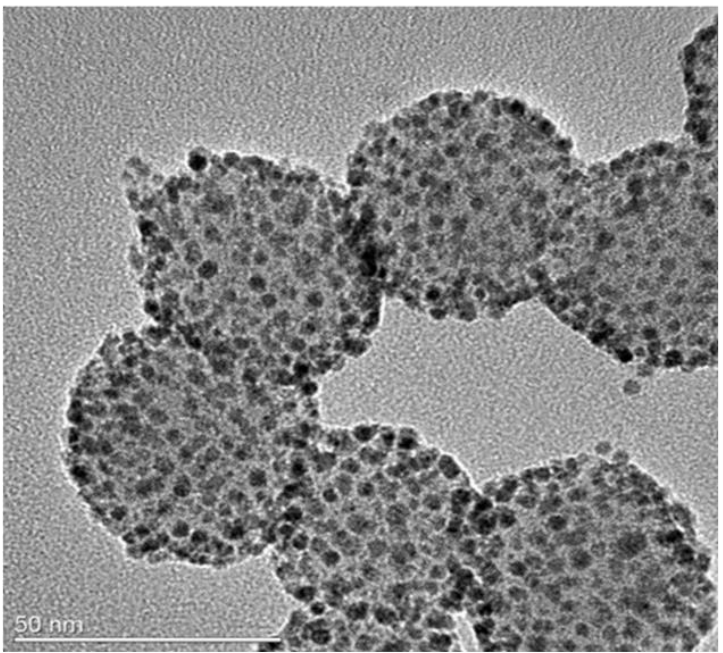

(c)

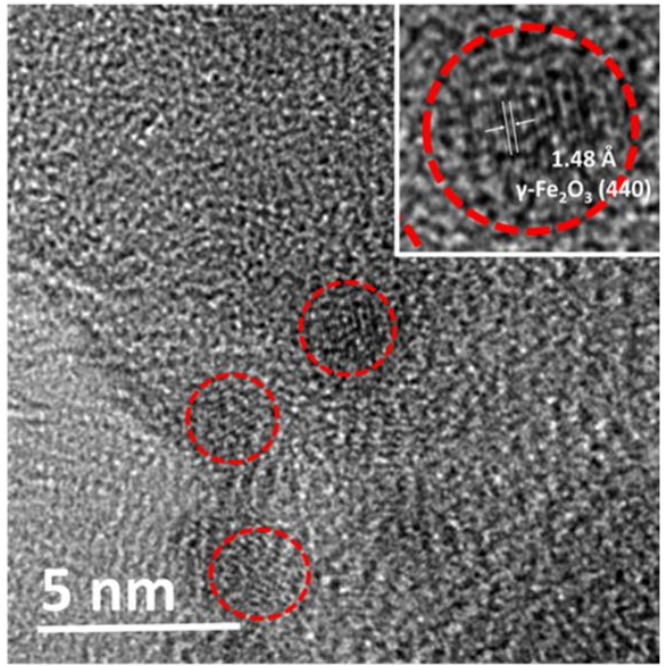

(d)

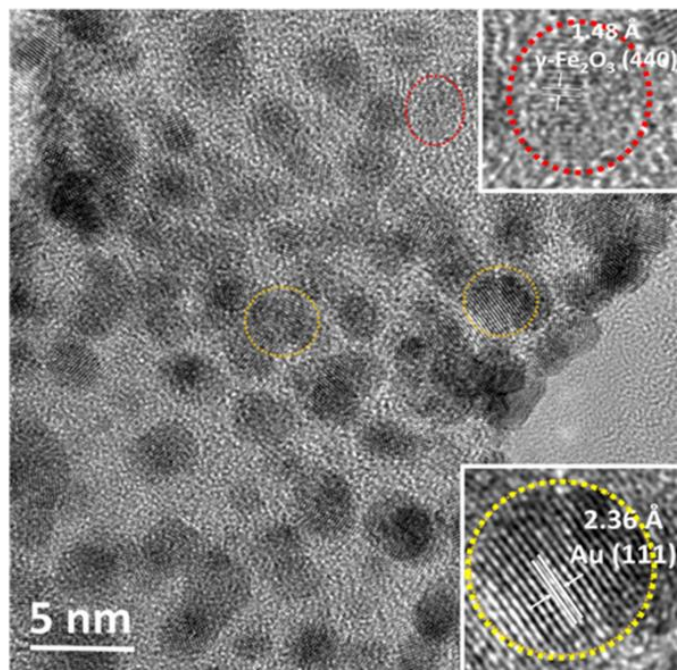

Figure S5. (a) SEM images of ligand-mS-ESIONPs@WMSNs. (b) TEM image of BAuNPs-ligand-mSESIONPs@WMSN. (c) The HRTEM image of ligand-mS-ESIONPs@WMSN. (d) The HRTEM image of BAuNPs-ligand-mS-ESIONPs@WMSN. 


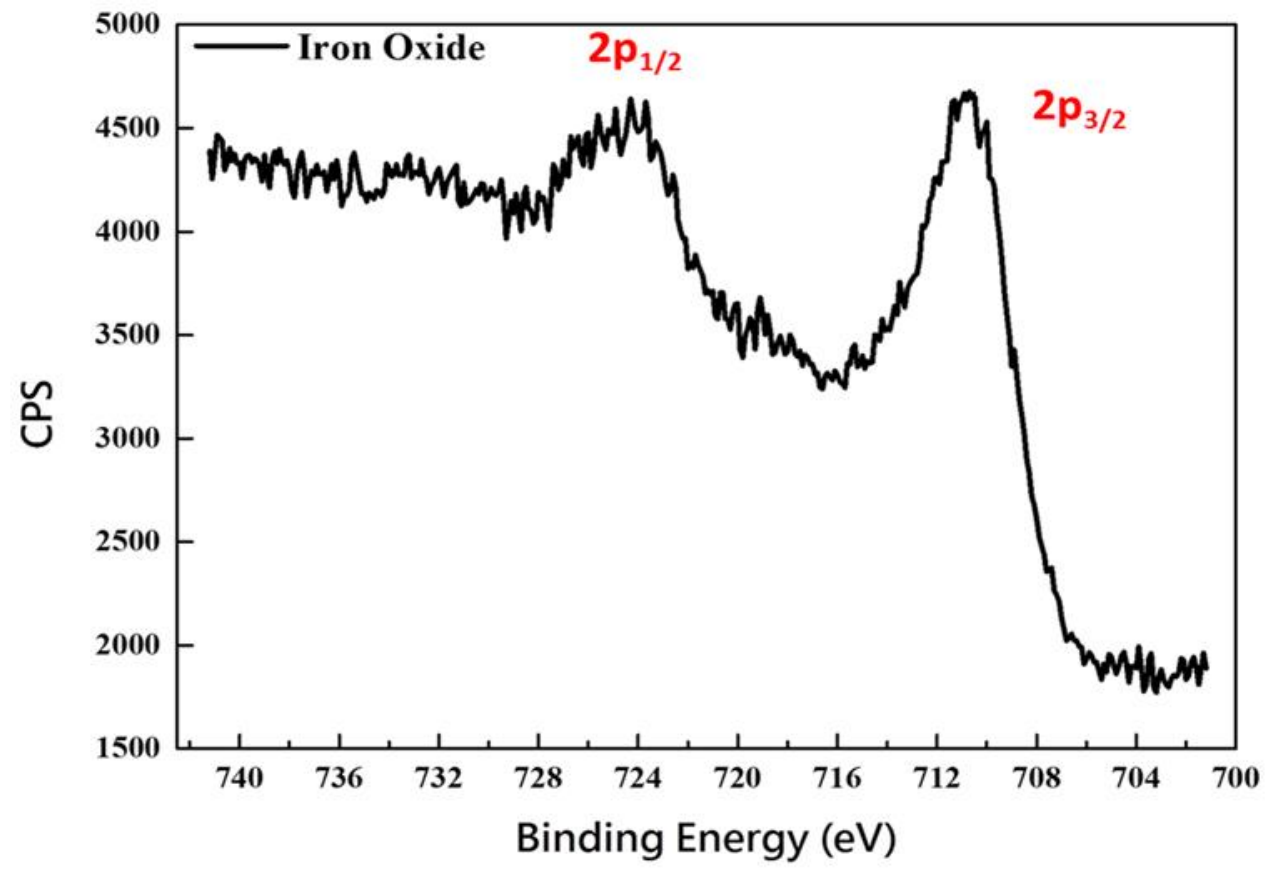

Figure S6. X-ray photoelectron spectroscopy (XPS) of ESIONPs. 

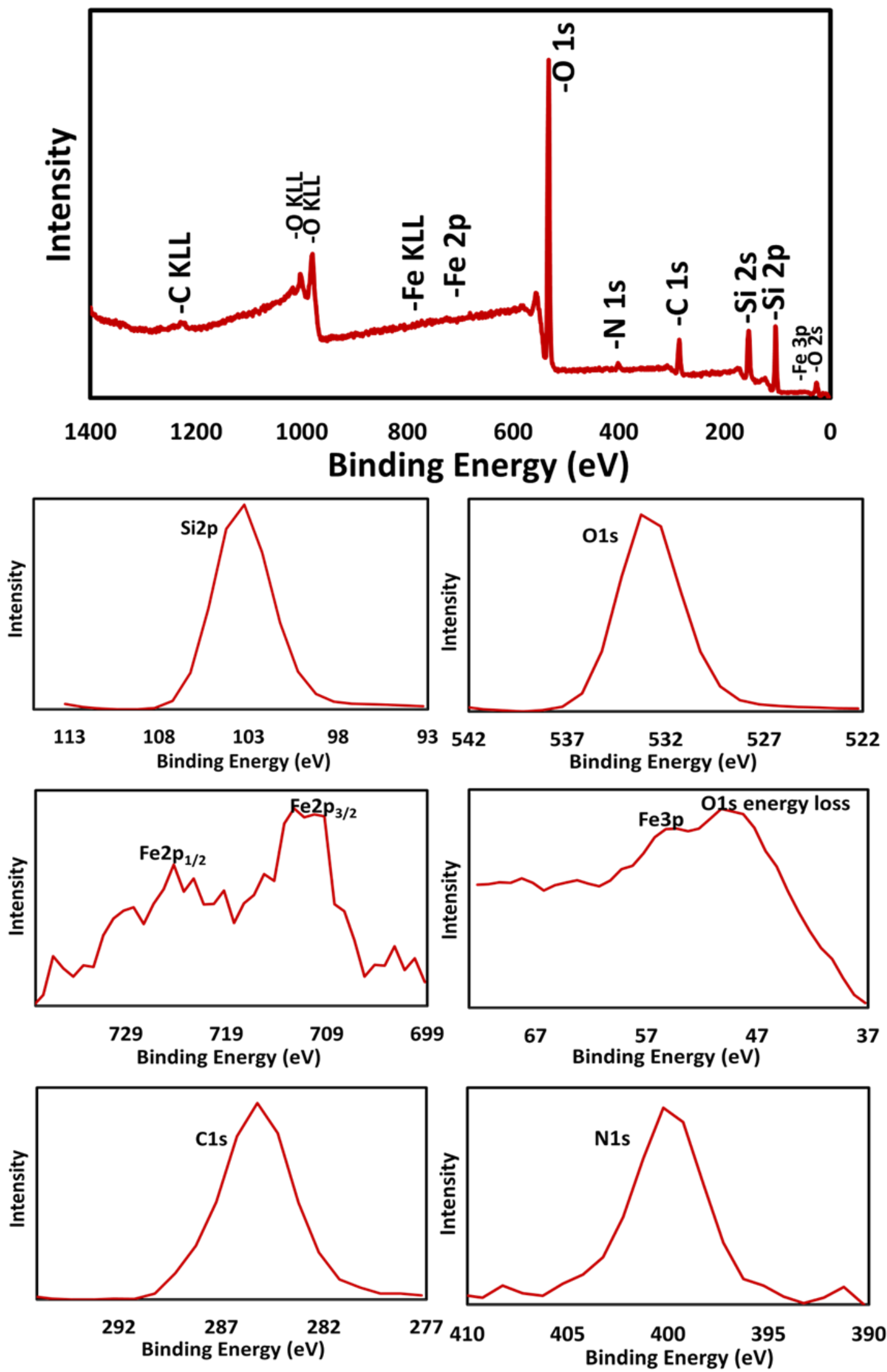

Figure S7. The full XPS spectrum of mS-ESIONPs@WMSN and the corresponding detailed spectra of major component elements. 


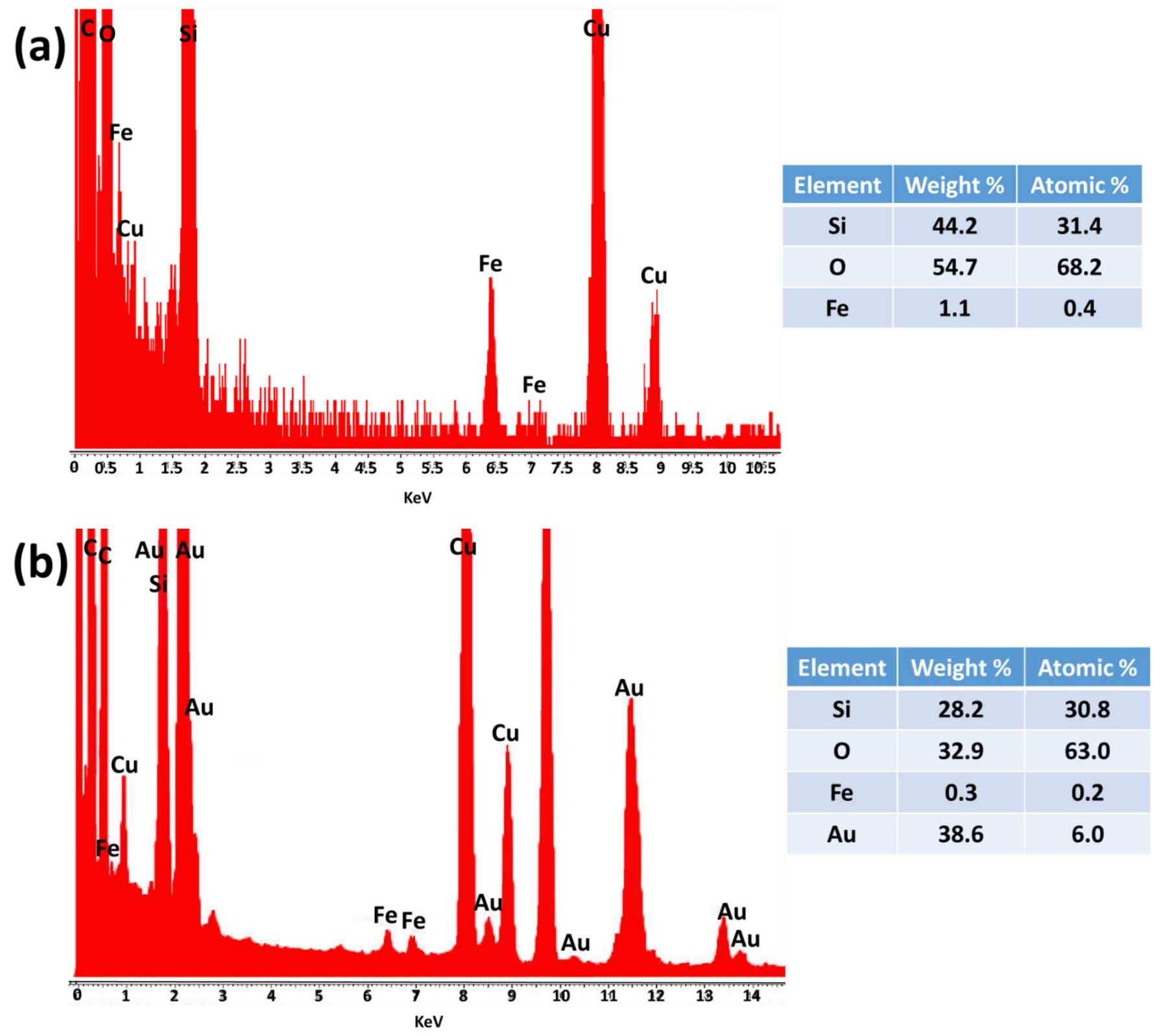

Figure S8. Energy-dispersive X-ray (EDX) spectroscopy analysis of (a) mS-ESIONPs@WMSNs, (b) BAuNPs-ligand-mS-ESIONPs@WMSNs. 

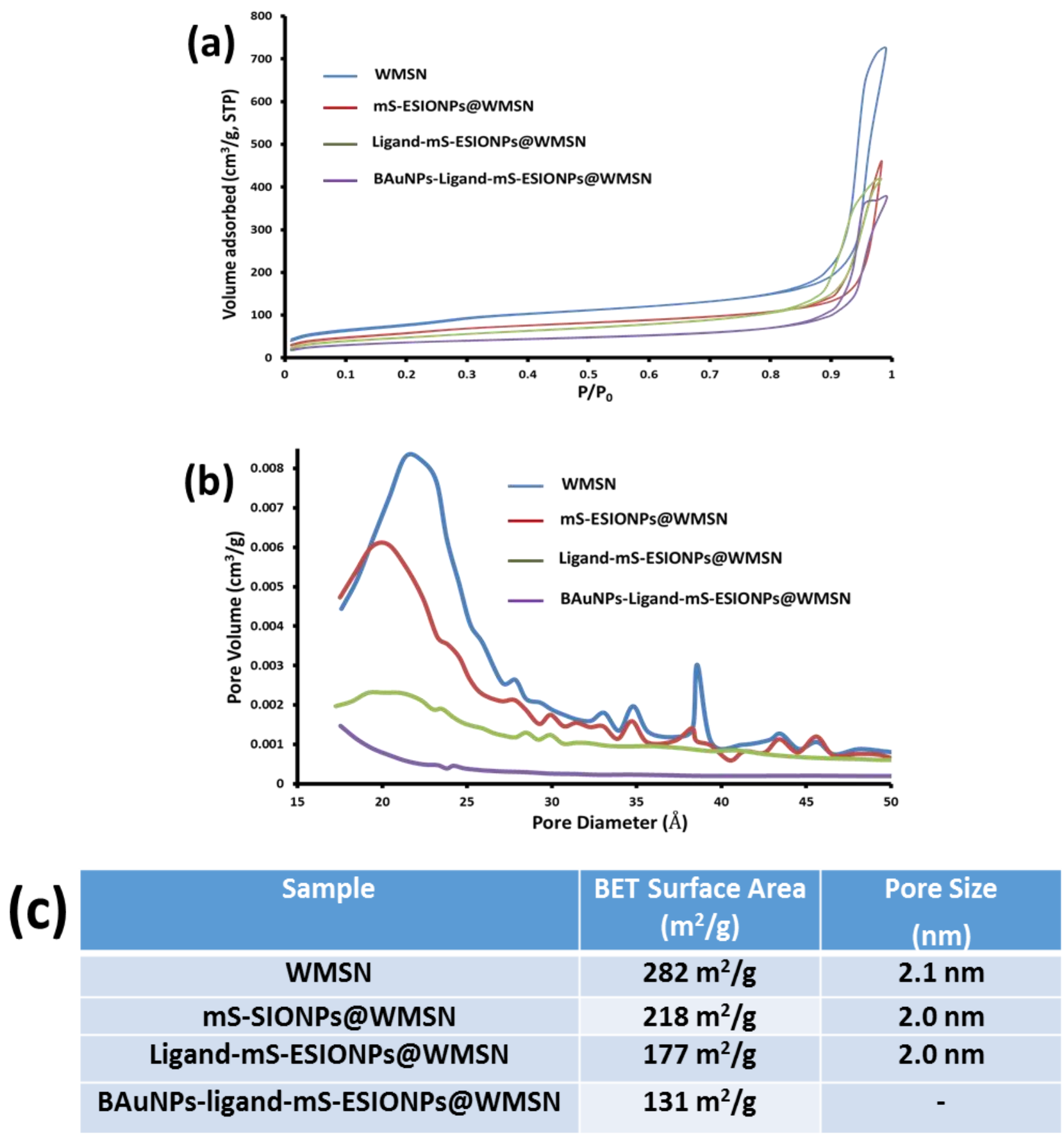

Figure S9. (a) $\mathrm{N}_{2}$ adsorption-desorption analysis (BET) of WMSNs, mS-ESIONPs@WMSNs, ligandmS-ESIONPs@WMSNs and BAuNPs-ligand-mS-ESIONPs@WMSNs. (b) pore-size distribution (BJH) of WMSNs, mS-ESIONPs@WMSNs, ligand-mS-ESIONPs@WMSNs and BAuNPs-ligand-mSESIONPs@WMSNs. (c) BET surface area and pore size calculated from $\mathrm{N}_{2}$ adsorption-desorption isotherm for WMSNs, mS-ESIONPs@WMSNs, ligand-mS-ESIONPs@WMSNs and BAuNPs-ligand-mSESIONPs@WMSNs. 


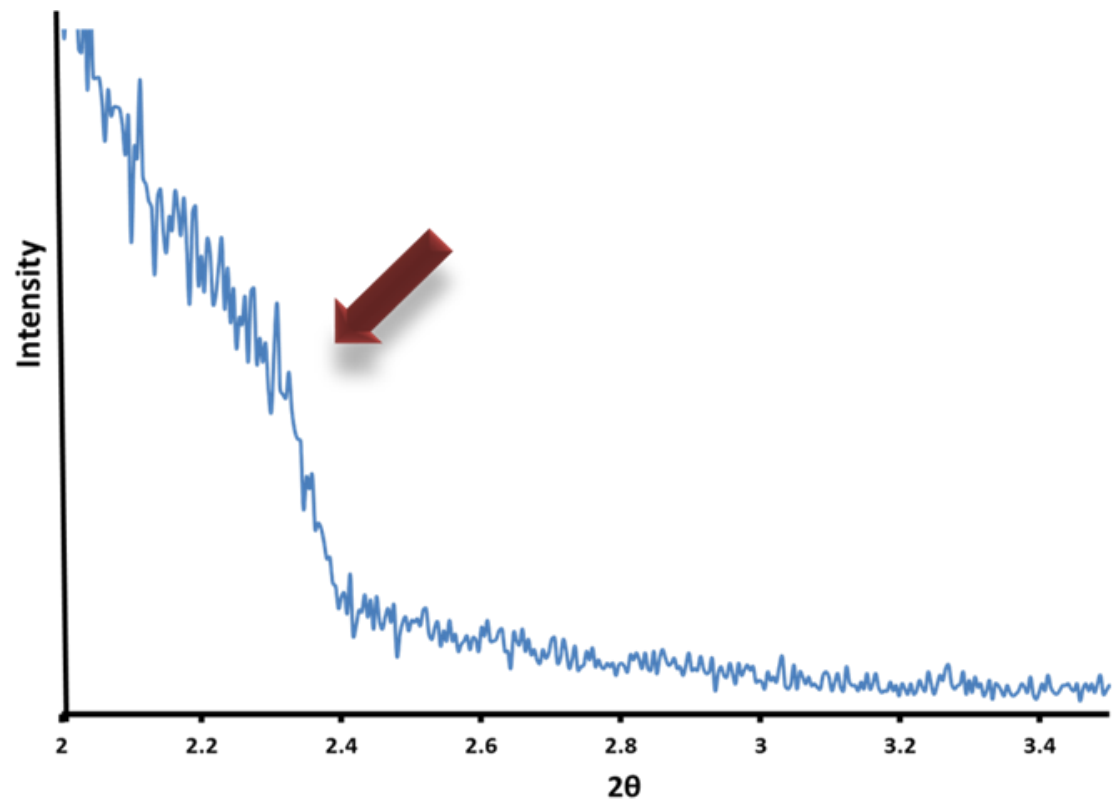

Figure S10. Small-angle X-ray diffraction pattern of WMSNs. The arrow indicates the diffraction signal from ordering mesoporous silica. 

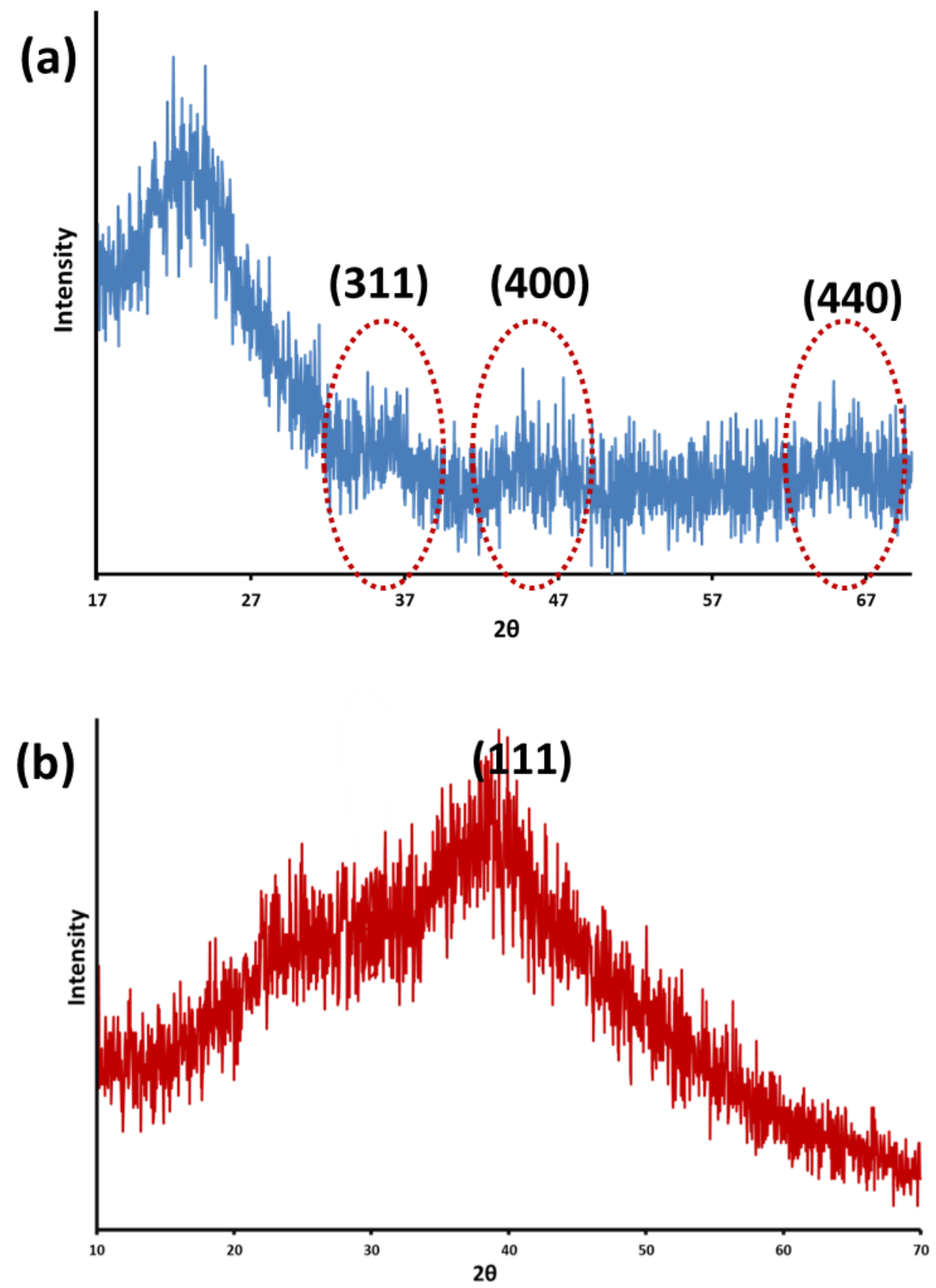

Figure S11. X-ray diffraction spectra of (a) mS-ESIONPs@WMSNs, (b) BAuNPs-ligand-mSESIONPs@WMSNs. 

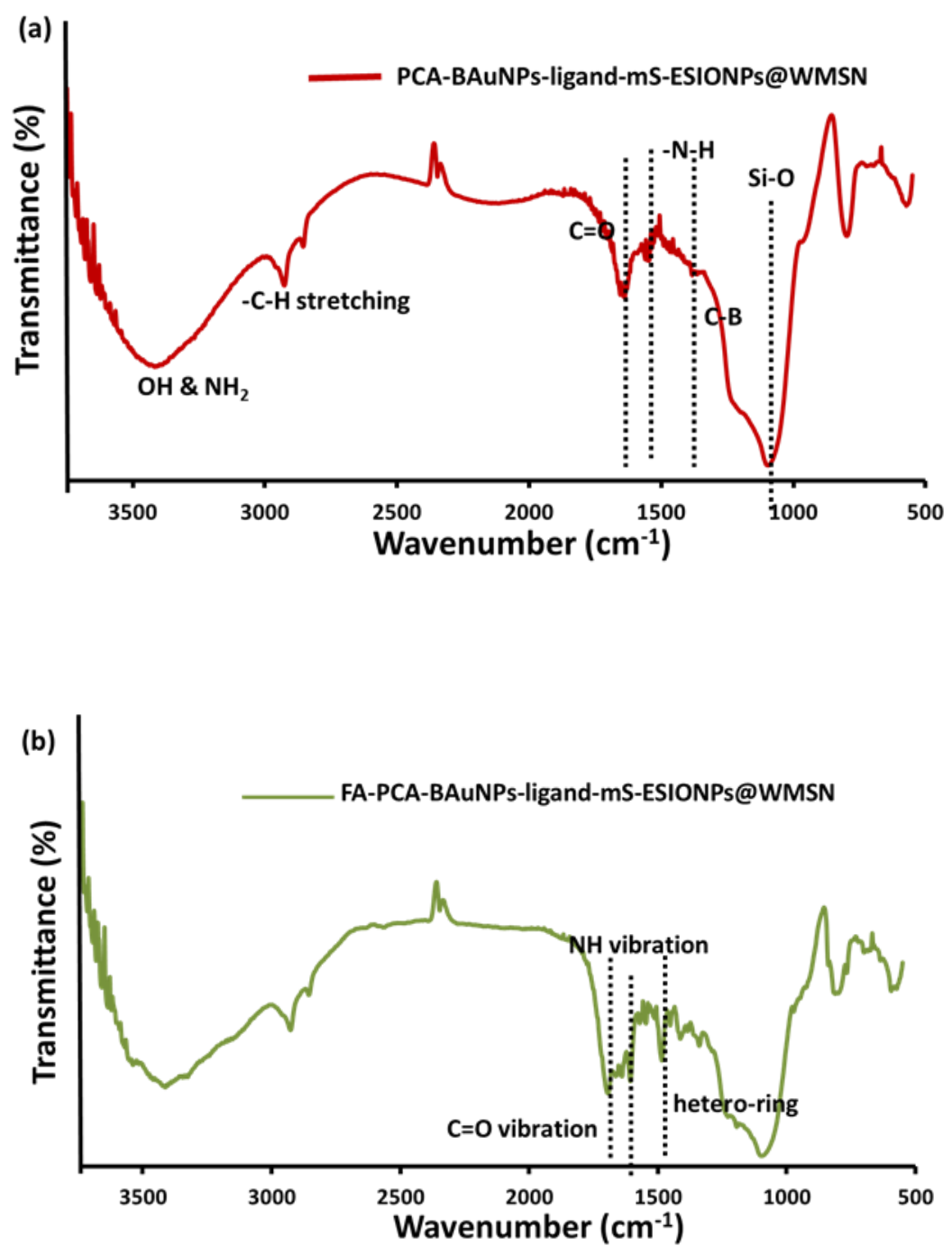

Figure S12. FT-IR spectra of FA-PCA-BAuNPs-ligand-mS-ESIONPs@WMSNs. The spectrum of FAPCA-BAuNPs-ligand-mS-ESIONPs@WMSNs exhibits the characteristic peaks of FA at 1643, 1604, and $1503 \mathrm{~cm}^{-1}$ corresponding to $\mathrm{C}=\mathrm{O}$ vibration and/or benzene (conjugated double bonds), $\mathrm{N}-\mathrm{H}$, and heteroring (conjugated double bonds), respectively. 


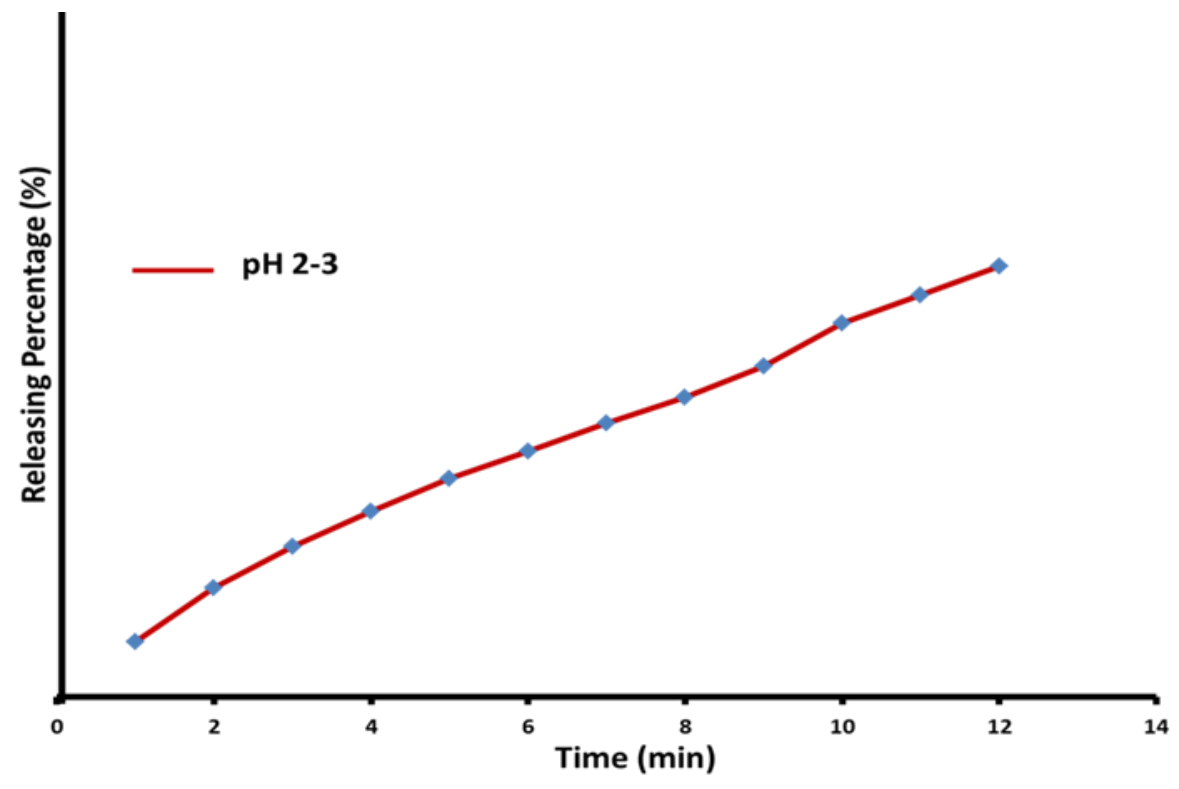

Figure S13. Release profiles of Safranin O dye in water from BAuNPs-ligand-mS-ESIONPs@WMSNs at $\mathrm{pH} 2-3$. 

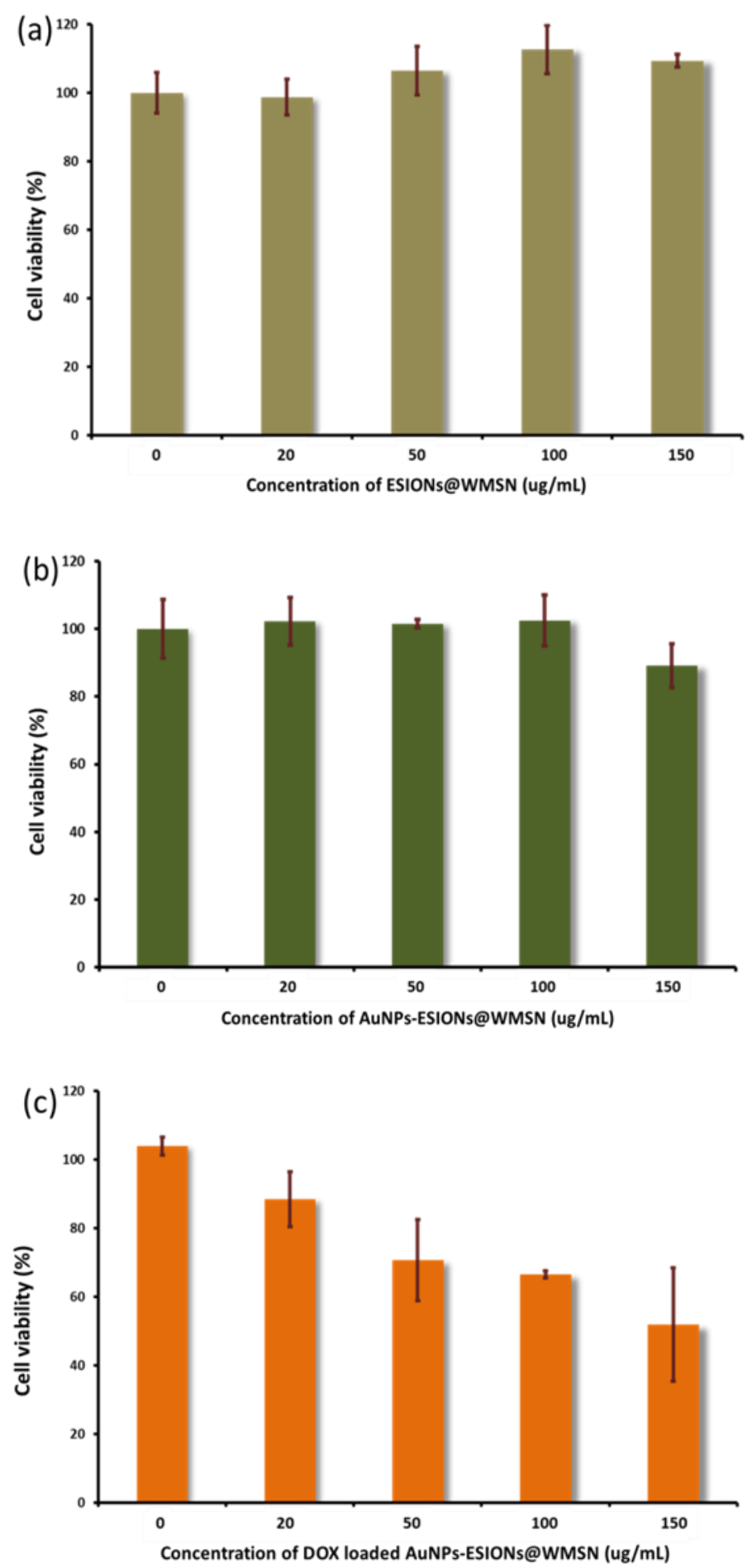

Figure S14. (a) MTT Assay of HeLa cells treated with ligand-mS-ESIONPs@WMSNs ranged from 0 to $150 \mu \mathrm{g} / \mathrm{mL}$. (b) MTT Assay of HeLa cells treated with BAuNPs-ligand-mS-ESIONPs@WMSNs ranged from 0 to $150 \mu \mathrm{g} / \mathrm{mL}$. (c) MTT Assay of HeLa cells treated with DOX loaded BAuNPs-ligandmS-ESIONPs@WMSNs ranged from 0 to $150 \mu \mathrm{g} / \mathrm{mL}$. 


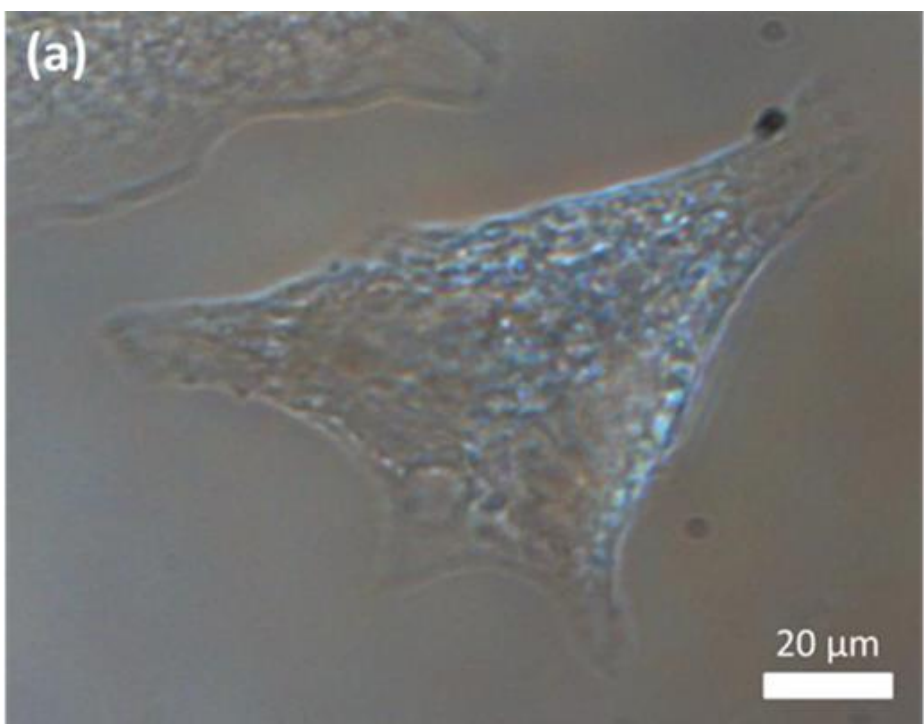

\begin{tabular}{|c|c|c|c|}
\hline Sample & $r_{1}\left(m^{-1} S^{-1}\right)$ & $r_{2}\left(m^{-1} S^{-1}\right)$ & $r_{2} / r_{1}$ \\
\hline ESIONPs & 3.54 & 11.29 & 3.19 \\
\hline $11.57 \mathrm{ppm} \mathrm{Fe}$ & 2.32 & 11.47 & 4.94 \\
\hline $12.95 \mathrm{ppm} \mathrm{Fe}$ & 3.00 & 13.67 & 4.55 \\
\hline $16.59 \mathrm{ppm} \mathrm{Fe}$ & 4.37 & 17.99 & 4.12 \\
\hline
\end{tabular}

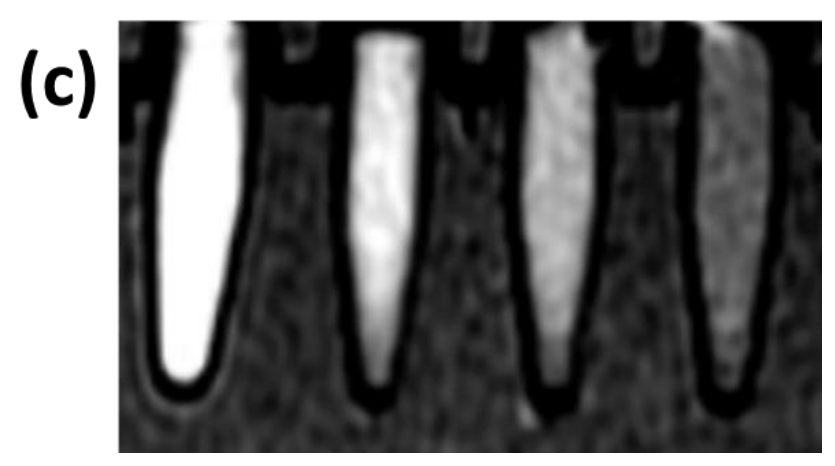

Figure S15. (a)Prussian blue stained images of BAuNPs-ligand-mS-ESIONPs@WMSNs treated HeLa cells. (High magnification images). (b) Relaxation properties of the nanocomposites at 0.47 T. ESIONPs refer to extremely small iron oxide nanoparticles and the other samples refer to ligand-mSESIONPs@WMSNs with different iron content. (c) $\mathrm{T}_{1}$-weighted images of BAuNPs-ligand-mSESIONPs@WMSNs with serial dilutions. From left to right: $[\mathrm{Fe}]=0.24,0.12,0.06,0.03 \mathrm{mM}$. 


\section{The estimation of the quantity of nanoparticles per cell.}

In our design, the drug delivery system is based on the mesoporous silica as a core. It is thus reasonable to assume that independent of functionalization on the surface of mesoporpous silica, the number of final nanocomposites should be the same. Therefore, the quantity of nanoparticles could be estimated by calculating the number of mesoporous silica cores. Because the ratio for iron oxide nanoparticles versus mesoporous silica cores could be controlled in the synthesis, the weight of mesoporous silica cores could be inferred according to the concentration of iron ions. As a result, the weight of mesoporous silica cores being ingested by cells could be deduced via the concentration of iron ion being measured by ICP-MS.

For example, in this study, comparing the iron concentration (measured by ICP-MS) in sample being fed with the amount ingested by cells, we obtain

$\frac{\mathrm{Fe}_{\text {ingested }}}{\mathrm{Fe}_{\text {feed }}}=\frac{M_{\mathrm{SiO}_{2} \text { ingested }}}{M_{\mathrm{SiO}_{2} \text { feed }}} \Rightarrow \frac{1.7 \mathrm{ppm}}{3.9 \mathrm{ppm}}=\frac{M_{\mathrm{SiO}_{2} \text { ingested }}}{6 \mathrm{mg}}, \therefore M_{\mathrm{SiO}_{2} \text { ingested }}=2.26 \mathrm{mg}$

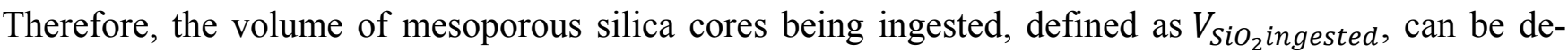
duced by

$V_{\mathrm{SiO}_{2} \text { ingested }}=\frac{M_{\mathrm{SiO}_{2} \text { ingested }}}{\rho_{\mathrm{SiO}_{2}}} \Rightarrow \frac{2.26 \times 10^{-3} \mathrm{~g}}{2.2 \mathrm{~g} / \mathrm{cm}^{3}} \quad, \therefore V_{\mathrm{SiO}_{2} \text { ingested }}=1.19 \times 10^{-3} \mathrm{~cm}^{3}$

The volume of an individual $50 \mathrm{~nm}$ mesoporous silica, defined as $V_{\mathrm{SiO}_{2}}$ single, can be calculated by $V_{\text {SiO }_{2} \text { single }}=\frac{4}{3} \pi \times\left(25 \times 10^{-7}\right)^{3}=6.54 \times 10^{-17} \mathrm{~cm}^{3}$

Thus, the number of nanoparticles being ingested, $N$, can be calculated by

$\mathrm{N}=\frac{V_{\mathrm{SiO}_{2} \text { ingested }}}{V_{\mathrm{SiO}_{2} \text { single }}}=\frac{1.19 \times 10^{-3}}{6.54 \times 10^{-17}}=1.82 \times 10^{13}$

The amount of nanoparticles per cell can then be estimated by dividing the number of nanoparticles with the number of cells (by cell counting),

$\frac{\mathrm{N}}{\text { cell }}=\frac{1.82 \times 10^{13}}{4 \times 10^{6}}=4.55 \times 10^{6} \# /$ cell 
(a)
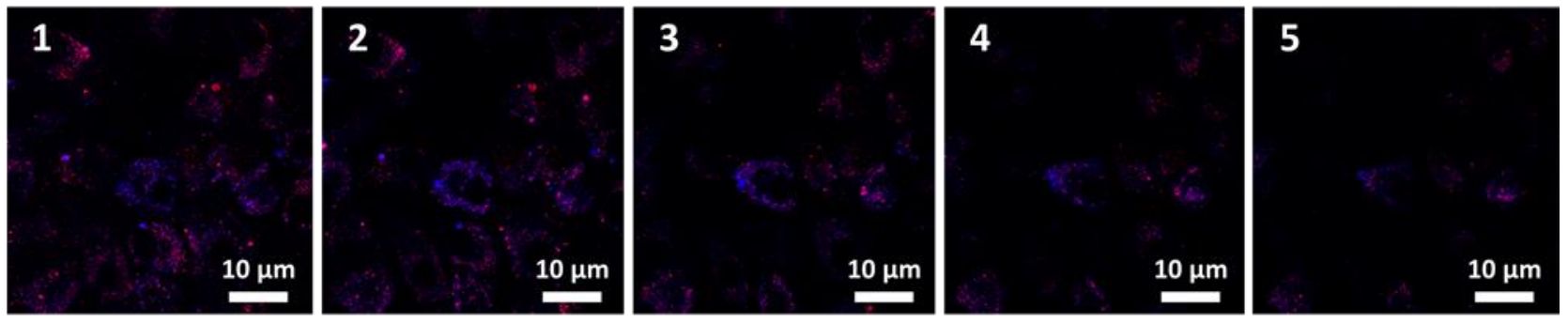

(b)
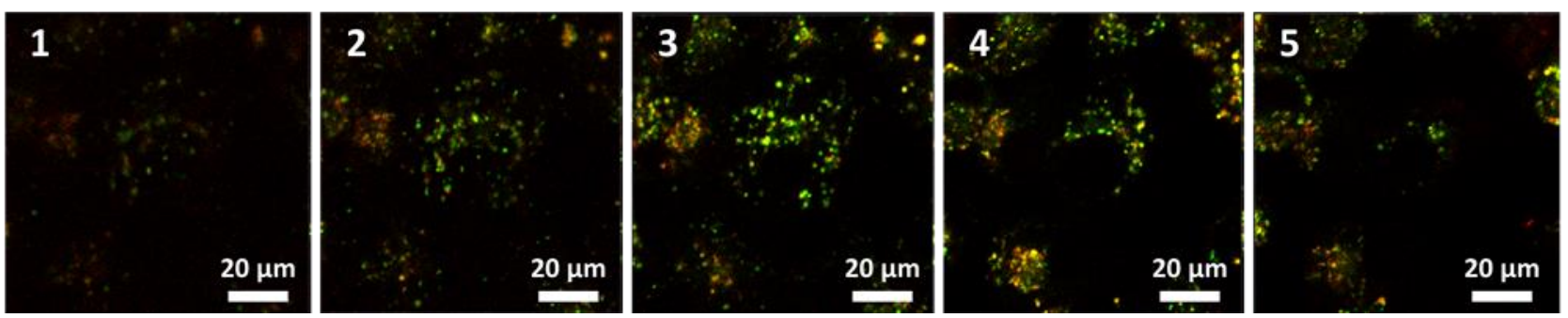

Figure S16. Serial sections of Z-stack confocal microscopic images of HeLa cells treated with FAPCA-BAuNPs-ligand-mS-ESIONPs. The endosomes and lysosomes were stained with (a) LysoTracker Blue and (b) Lysotracker Green. The merged image represents LysoTracker + DOX emission. 


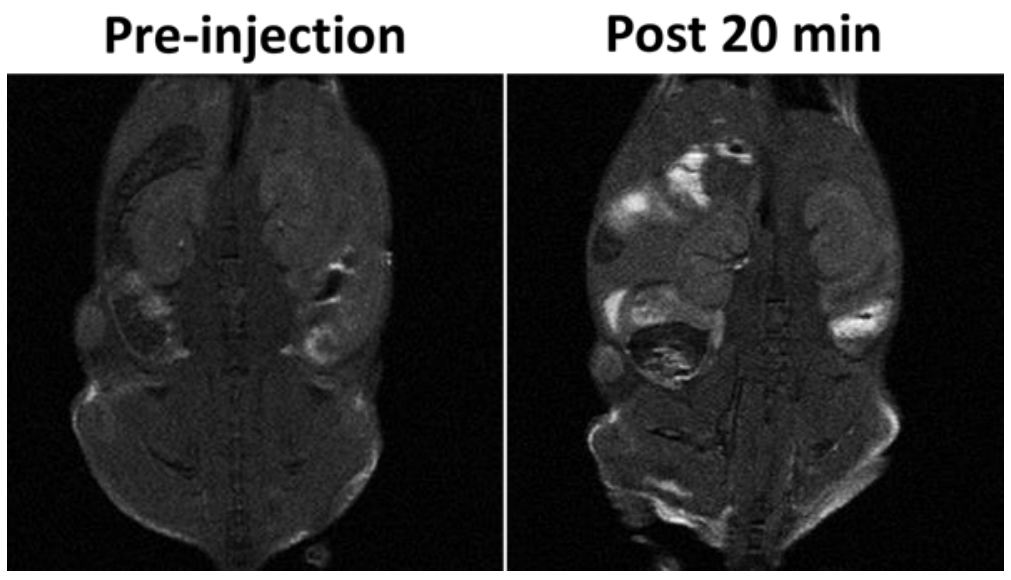

Figure S17. The $\mathrm{T}_{1}$-weighted images of FA-PCA-BAuNPs-ligand-mS-ESIONPs@WMSNs were monitored using a 7T animal micro MRI system as the NPs were administrated by intravenous injection of 1 $\mathrm{mg} \mathrm{Fe} / \mathrm{kg}$. The images were captured before and 20 minutes after intravenous injection. 


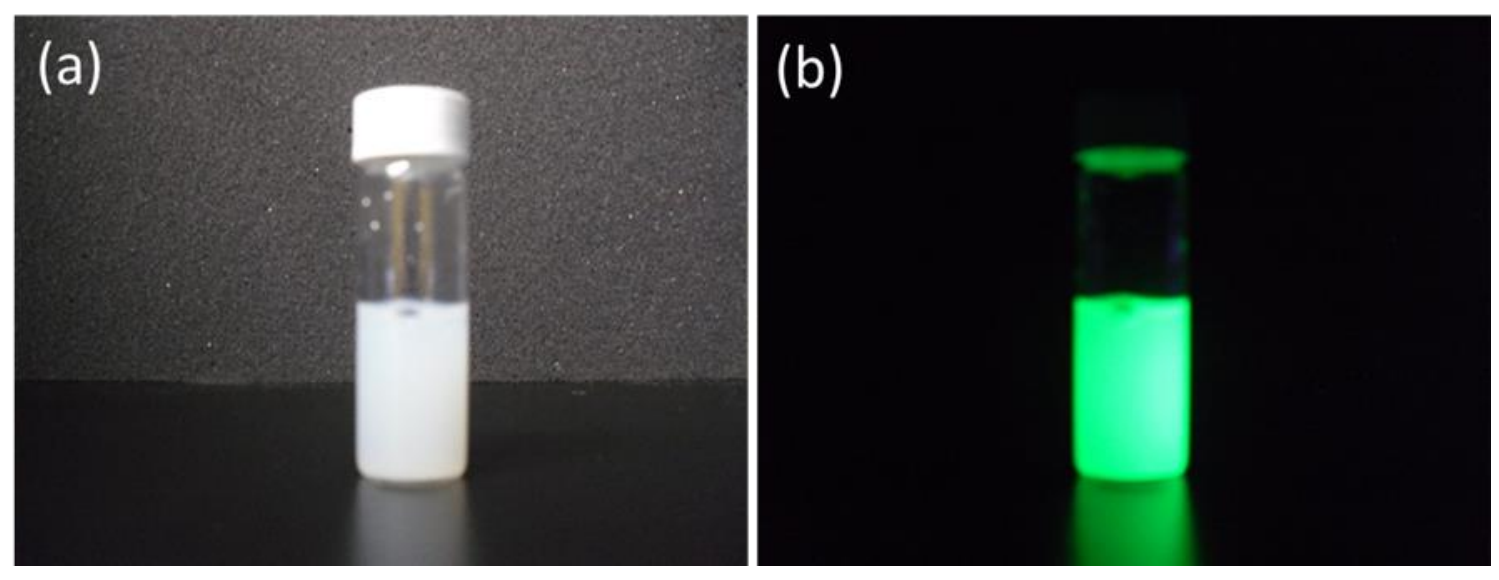

Figure S18. Using the same procedure of adsorbing nanoparticles on the surface of wormlike mesoporous silica and then coated with a thin layer of mesoporous silica shell to encapsulate quantum dots within WMSN. (a) The as-synthesized nanoparticles. (b) Particles under UV lamp excitation source. This experiment aimed to demonstrate that this method can also be used to encapsulate nanoparticles other than ESIONPs. 


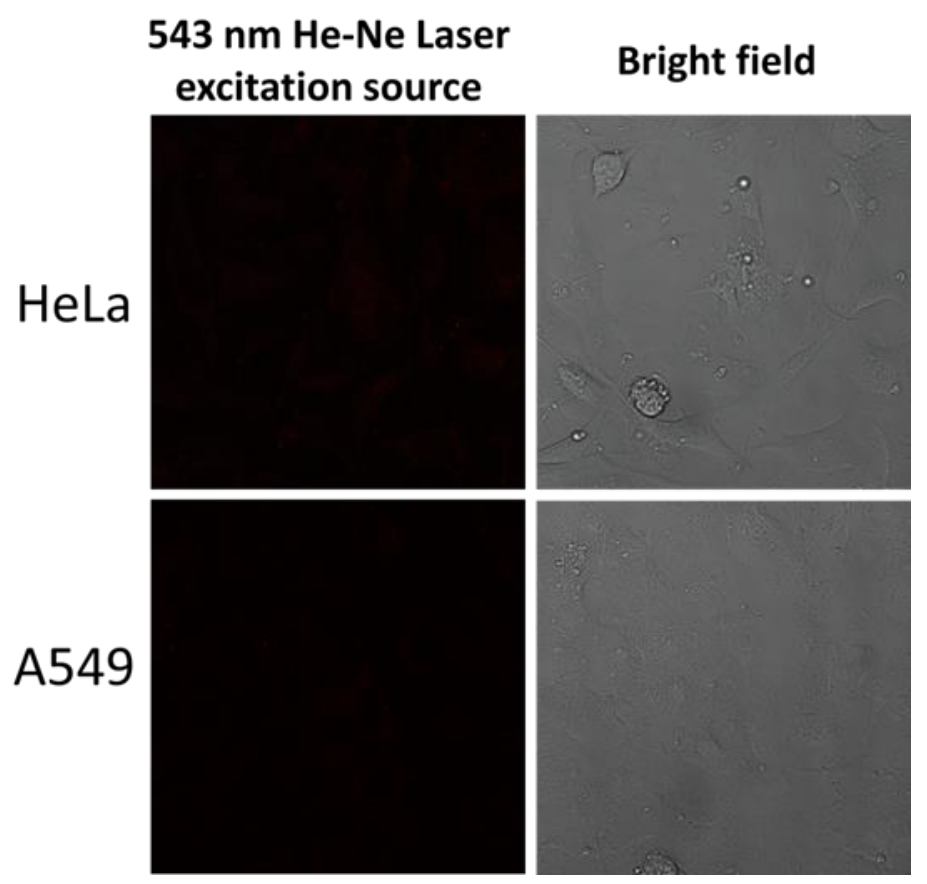

Figure S19. Confocal images of control set for HeLa and A549 cell lines without being fed with doxorubicin. We used $543 \mathrm{~nm} \mathrm{He-Ne} \mathrm{laser} \mathrm{as} \mathrm{the} \mathrm{source} \mathrm{to} \mathrm{ensure} \mathrm{the} \mathrm{absence} \mathrm{of} \mathrm{autofluorescence} \mathrm{under} \mathrm{ex-}$ citation and the emission intensity in Figure 5 and Figure 6 indeed came from Dox. 